of at least some of their terrors and disastrous results. located. The ununited fracture of the clavicle was more or With this serum as with serum therapy in general the earlier it is used the better the results attained.

126 State Street.

\section{RESECTION OF TWO INCHES OF THE HUMERUS AND WIRING, WITH A REPORT OF A CASE OF WIRING OF THE CLAVICLE FOR UN. UNITED FRACTURE.}

BY STEWART I. MCCURDY, A.M., M.D. Orthopedic Surgeon to Presbyterian Hospital, Member Americau Orthopedic, American Medical, and Allegheuy County Medical Societies; Professor Anatomy and Oral Surgery, P'ittsburs Dental College, etc.
PITTSB URG, PA.

It is a matter of selection, after excision of joints or resection of bones, whether nails are selected to secure the bones, or whether wire is used.

Possibly I am stating the facts, when the statement is made that the majority of surgeons prefer spikes to wire. Especially is this true for excisions of the knee.

It is not the adaptation of bones after excisions for chronic tubercular disease, I desire to consider, but rather the use of wire for the treatment of compound and irreducible fractures.

Fia. 1 .

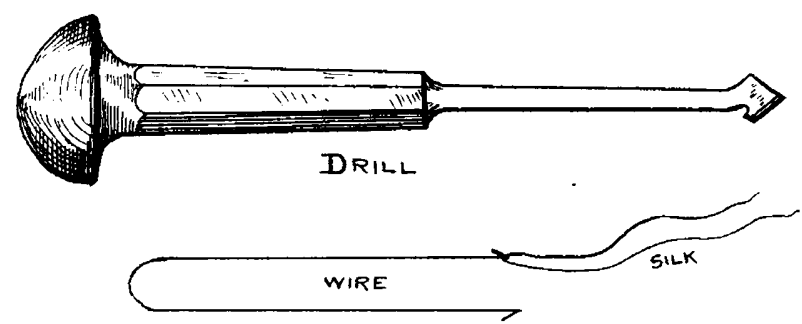

That it is possible for me to secure a more perfect adaptation of bones by the use of wire goes without further discussion. That other operators can not get as perfect results with wire and use spikes instead, also goes with saying. Whether these men have tried wiring and discarded it for the nails, $I$ am unable to say. To attempt to do a bone wiring without the proper instruments would be as difficult as to do a vaginal hysterectomy without an equipment.

To Dr. Wm. F. Fluhrer of New York, must be given the credit for perfecting instruments for bone wiring. In order that bone wiring might be speedily done it is necessary to use a notched drill. It is an easy matter to pass a drill through a bone, but it is not such an easy matter to keep the drill hole pervious or reënter again after the drill has once been withdrawn.

As will be observed the drill is notched near the point. After it passes through the bone a silk thread is thrown around the point and allowed to engage in the notch, after which it is withdrawn. The drill hole once loaded with silk, the wire is attached and drawn through either way as desired.

Case 1.-Mr. P. F., aged 35, was thrown from a carriage and received a dislocation of the left shoulder and fracture of the left clavicle. After reduction of the dislocation the shoulder recovered promptly. The displacements of the fragments in the fractured clavicle were so great that no effort was made by nature to repair the injury. About six months after the original injury he was again injured and the shoulder was dis- less disturbed.

The fracture was painful at all times when the arm or shoulder was moved. The internal end of the external fragment appeared to be sharp and the patient, if he had had the

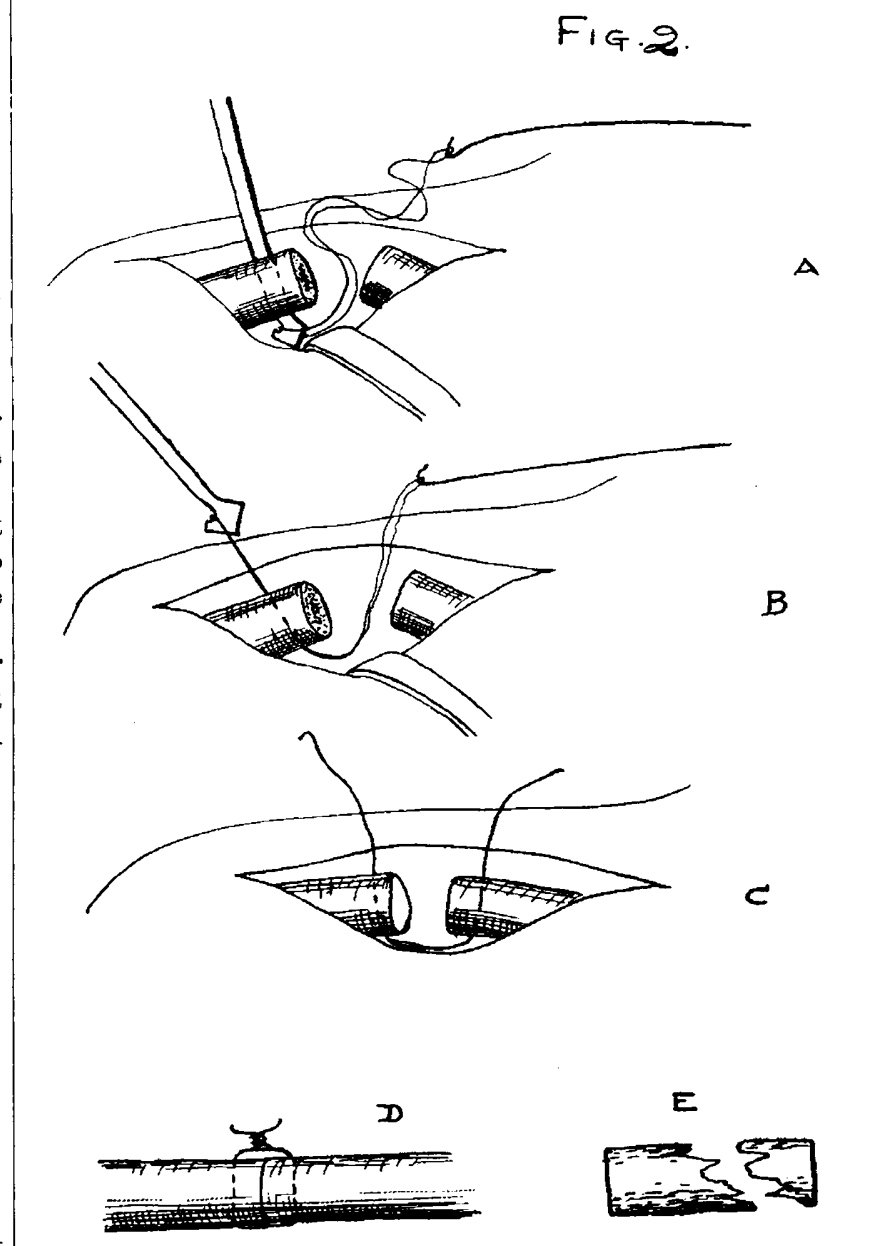

power in the arm, could not work on account of the pain caused by the motion.

A study of the anatomy of the parts (Fig. 3) shows that the free end of the under fragment was pushed down on the tissues overlying the subclavical artery and brachial plexus, when

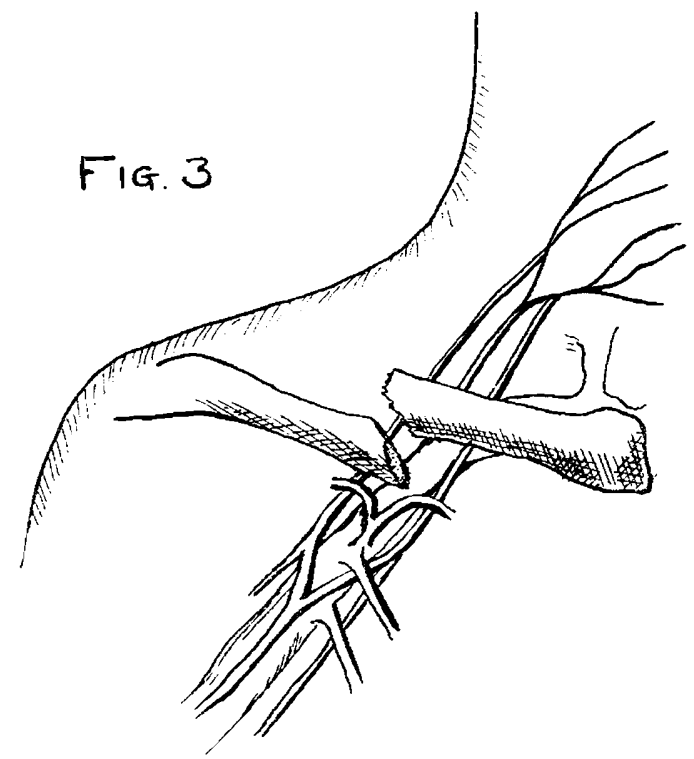

even the arm was elevated. Operative interference was advised for two reasons: 1, that union of the bones might be 
secured; 2 , that the pain caused by the projecting end of the bone might be relieved.

Operation: Free longitudinal incision was made along the clavicle and the ends were dissected out. The ends were sawed off by the use of a chain saw. The bones were drilled and brought together with silver wire.

Result: Union was perfect, and the wound healed without suppuration. The arm is practically as good as before the injury. The wire was subsequently removed.

Case 2.-H. B., aged 30, brakeman, received an injury of his left thumb while making a coupling. The muscles of the palmar surface of the thumb were squeezed through the skin, and the bone was broken; the thumb was practically torn off. Preparation was made to amputate. While my assistant was

\section{FiG 4.}

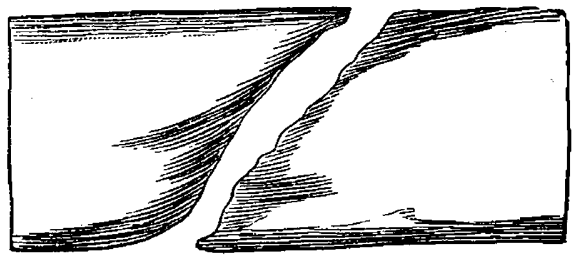

anesthetizing the patient, it occurred to me that wiring might save the thumb, and the latter operation was done. In six weeks the wound had entirely healed and in three months the thumb was as well as before the accident, save some limitation in extension as a result of the extensive laceration of the muscles. This was my first wiring and was done in June, 1885.

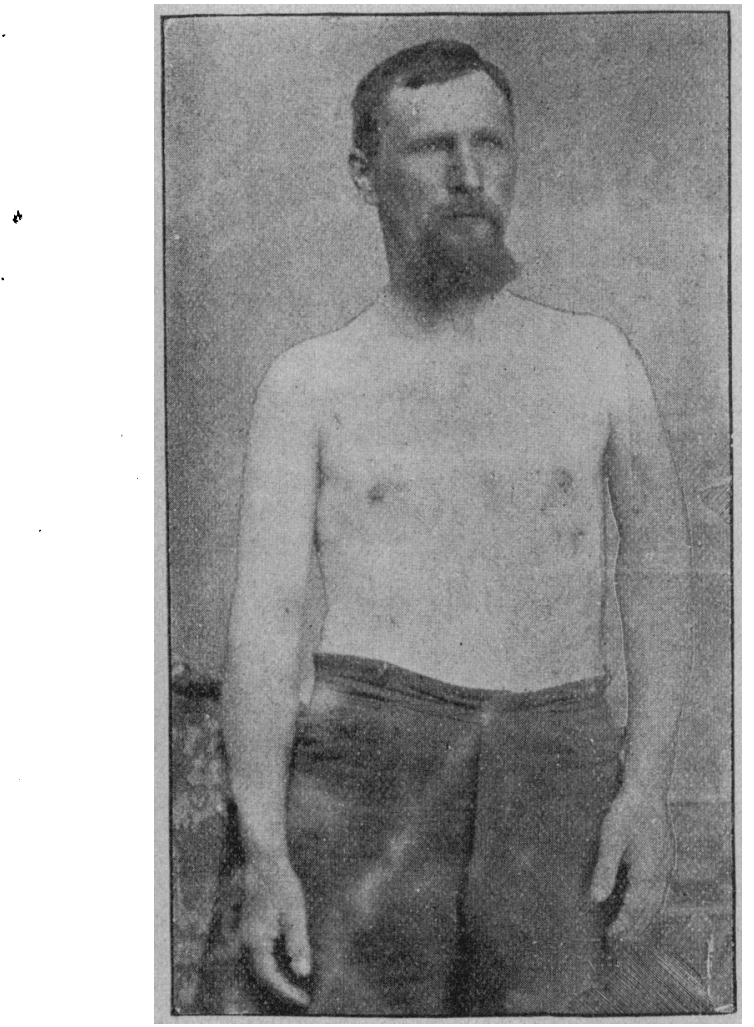

FigURE 5.

Case 3.-D. W. C., aged 37 years. The contents of a shotgun had passed through the arm internally to the thickest portion of the biceps and making its exit near the insertion of the deltoid. The humerus was entirely torn off; the patient favored amputation at my first visit, but we concluded to adjust fracture and wait for results.

The bones showed no signs of union, and the case was complicated by a severe hemorrhage three weeks after the injury, and at this time the patient insisted upon amputation. I assured him that amputation was not demanded, but instead I would advise excision and wiring, which operation was done, three weeks after the injury.

Two inches of bone were removed (Fig. 4), and the bone adjusted the wire. The ends of the bone were ragged and showed no evidence of repair.
The case from the date of wiring did not have an unfavorable symptom, and in six weeks was entirely healed.

The man is a farmer and has made a full hand at every kind of work ever since. The two inches of shortening did not interfere with this in the least. (See Fig. 5.)

Description of cuts: Fig. 1 shows notched drill, the wire and silk and the method of attaching them. Fig. 2 shows, diagrammatically, the method of loading the drill hole with the wire as the drill is withdrawn, as well as other steps in wiring. Fig. $2 \mathrm{E}$ shows the bone removed in the clavicle case.

\section{SELECTIONS.}

The French National Population Alliance.-The London Times states that an organization, with medical men at its head, has been formed in Paris to stay the French population's decline. The organizers are Dr. Jacques Bertillon, chief of the bureau of statiatics of Paris, Dr. Charles Richet, professor of the faculty of medicine in the same city, and Dr. Javal, member of the Academy of Medicine. For years these men have noticed with regret that the population of France was not increasing proportionately with the population of other countries, and now they come forward with what they believe to be an infallible remedy for this evil. That some drastic remedy is needed they insist, as otherwise France must soon sink to the rank of a second rate, or even a third rate nation. A hundred years ago the great countries, in other words, the great powers of Europe contained $98,000,000$ inhabitants, of whom $26,000,000$, or 27 per cent., were residents of France. To-day these same countries contain $300,000,000$ inhabitants, of whom $38,000,000$, or only 12 per cent., live in France. These figures speak for themselves, and the obvious conclusion, according to Dr. Ber tillon and his colleagues, is that if the births continue to decrease at the same ominous rate, France, which was once one of the most powerful countries in Europe, will soon be one of the weakest. These gentlemen have begun their novel campaign by founding a society which is styled "The National Alliance for the Relief of the French Population." The defensive measures which they propose to adopt are numerous, but for the immediate present they will confine their attention to three points: 1 , they will try to get the laws relating to inheritances greatly modified; 2 , they will try to get all direct taxes removed from those families which have more than three children, and 3 , they will try to have the laws relating to succession duties thoroughly reformed. As it is manifest that the nation's treasury would be seriously affected by the removal of taxes in the case of all families which have more than three children, it is proposed to place a tax of 1-5 per cent. on all families which are childless, or have only one or two children. There is said to be excellent reasons for these proposed reforms. The larger a man's family is in France, we are told, the more he is taxed, both directly and indirectly, with the result that thrifty parents do not care to have many children. Moreover, the parents' property must be divided among the children, and if the children are numerous and the property small, the disastrous results can easily be foreseen. In other countries the law of entail is maintained pretty rigidly and though its operation seems hardly equitable or natural, as regards younger children, it certainly seems to act in some degree as a preventive of depopulation. "Remove all burdensome taxes from fruitful families," say the members of the National Alliance, "and let Frenchwomen and Frenchmen know that even from a monetary point of view, it will be fortunate for them to have many children. Then as the national treasury must be supported, let us tax those adults who have few or 